\title{
Caracterización de la Cultura Organizacional Dominante en las Empresas Familiares de Servicios de Ciudad Victoria Tamaulipas
}

\section{Daniela Cruz Delgado, Estela Torres Ramírez1}

Universidad Politécnica de Victoria

\section{Recepción: 30 de Septiembre del 2016 \\ Aceptación: 28 de Octubre del 2016 \\ Publicación: 16 de Diciembre del 2016}

El análisis de la cultura organizacional se ha convertido en elemento central en las organizaciones de todo tipo. Su vínculo con la empresa familiar es fundamental para comprender la conducta de los integrantes de este tipo de empresas cuyas características particulares las hacen propias. El objetivo de este estudio fue identificar el tipo de cultura organizacional dominante, actual y preferida, en las empresas familiares de servicios de Ciudad Victoria, Tamaulipas. El modelo utilizado fue el propuesto por Cameron y Quinn (1999), el cual distingue cuatro dimensiones de la cultura organizacional: clan, adhocrática, de mercado y jerarquizada. Se seleccionaron seis empresas de servicios. La cultura organizacional actual y deseada identificada en las empresas familiares objeto de análisis fue la cultura tipo Clan. En la que los integrantes tienen un alto sentido de pertenencia a la organización y su interacción es como la de una gran familia. Un factor fundamental para la caracterización de la cultura organizacional predominante es el tiempo que llevan funcionando en el mercado. Las tres tipos restantes de la cultura organizacional presentaron cambios entre la actual y deseada, el cambio más notorio se dio entre la adhocrática y jerarquizada.

\section{Palabras clave: Adhocracia, valores organizacionales, jerarquía organizacional.}

CHARACTERIZATION OF THE DOMINATING ORGANIZATIONAL CULTURE IN THE FAMILY BUSINESSES OF THE SERVICES SECTOR IN CIUDAD VICTORIA, TAMAULIPAS ABSTRACT

The analysis of the organizational culture has become a key element in all kind of companies.
Its link with the family business is essential to understand the behavior of the members of these kind of companies which particular features made them unique

Profesora investigadora de tiempo completo en el Programa Académico de Administración y Gestión de Pequeñas y Medianas Empresas.

objective of this study was to identify the dominating, current and favorite kind of organizational culture in the family businesses of the services sector in Ciudad Victoria, Tamaulipas. The standard used is the one proposed by Cameron and Quinn (1999) that distinguish four different dimensions of the organizational culture: clan, adhocracy, market and hierarchy. Six companies of the service sector were selected. The clan was the chosen dimension of organizational culture for this analysis, in which the members have a high sense of belonging to the company and interact as a big family.

A fundamental factor for the characterization of the dominating organizational culture is the time they have been operating in the market. The other three dimensions of organizational culture presented changes between the current and most liked. The most notorious was between the adhocracy and the hierarchy. After the clan in the current organizational culture prevails the hierarchy. But the most liked is the adhocracy. This shows that the members of the studied companies do not wish to work and stablish relations inside a company in which a strict set of rules and procedures limits them to do what is asked from them or show them what and how to do their tasks. On the contrary, they like an organizational culture in which the company is a dynamic place to work and allowed them to explore their entrepreneurial spirit with ideas for the strengthening and growth of the company.

Keywords: adhocracy, organizational values, organizational hierarchy 
El desarrollo económico de los países requiere del eficaz funcionamiento de las entidades económicas que constituyen el sector empresarial, del cual las empresas familiares constituyen parte esencial. Estas unidades económicas tienen características particulares respecto a la empresa no familiar, uno de sus principales rasgos distintivos es la cultura organizacional.

El análisis de la cultura organizacional se ha convertido en elemento central en las organizaciones de todo tipo. Su estudio revela una aportación a la teoría del comportamiento organizacional. El vínculo con la empresa familiar es fundamental para comprender la conducta de los integrantes de este tipo de empresas cuyas características particulares las hacen propias de estudios que identifiquen áreas de oportunidad al respecto.

Las particularidades de cada organización se reflejan en su cultura, la cual está influida por la conducta y actitudes de su capital humano, los empleados, quienes con su comportamiento afectarán los resultados de la empresa en su conjunto y serán los determinantes del logro o no de los objetivos planteados.

La cultura organizacional es dinámica, cambia a largo plazo, se ajusta a las necesidades del contexto en el que se encuentra inmersa la organización. Si la empresa no cambia y se ajusta a las demandas del mercado perecerá. Si la empresa cambia y genera ventajas competitivas, permanecerá en el mercado. El cambio es inherente a la cultura organizacional, por lo que es importante que las organizaciones identifiquen sus características culturales y corroboren, previo análisis del entorno, si son las necesarias para su supervivencia.
La empresa familiar, como toda organización se enfrenta a patrones de conducta, valores y actitudes particulares, que representan su cultura, la cual suele ser muchas veces más arraigada y ello constituye la diferencia con las empresas no familiares.

Las definiciones de empresa familiar distinguen dos factores esenciales que la caracterizan: la participación de los integrantes de la familia y el tiempo que los mismos le dedican (Leach, 2009 citado por Garza et. al. 2011). La participación de los miembros de la familia se puede dar a cualquier nivel de la organización y el tiempo dedicado también varía entre los integrantes.

El vínculo entre cultura organizacional y empresa familiar se ha analizado desde diferentes perspectivas, entre las que se pueden mencionar la que analiza alguno de los elementos de la cultura organizacional en este tipo de empresas (Garza et. al. 2011; Steckerl, 2006), las aproximaciones teóricas que analizan la cultura empresarial en la gestión de la empresa familiar (Esparza, et. al. 2010) y los criterios usados para la definición del concepto (Barroso, et. al. 2012).

La cultura organizacional comprende los valores, las creencias, ideologías, hábitos y costumbres de los integrantes de la empresa, en la transmisión de esa cultura el emisor es un factor clave el para éxito organizacional, como lo manifiesta Cújar (2013). En la empresa familiar el emisor es el propietario. Esos elementos de la cultura organizacional generan conductas que benefician o perjudican a la empresa dependiendo de la atención que se les brinde. Por ejemplo, culturas organizacionales que incluyan entre sus elementos la adaptabilidad pueden ser propicias para la innovación (Gómez y Ricardo, 2012), elemento fundamental en el entorno globalizado en el que funcionan las organizaciones. 
Este estudio se justifica porque las empresas familiares son un tipo de empresas que prevalecen en Ciudad Victoria, Tamaulipas, las hay en el giro industrial, comercial y de servicios, el mayor porcentaje se ubica en los dos últimos. Se eligió el giro de servicios por ser un sector de alto valor agregado para la economía regional y nacional.

En términos generales, el objetivo de este estudio fue identificar el tipo de cultura organizacional dominante actual y preferida en las empresas familiares de servicios de Ciudad Victoria, Tamaulipas, con el propósito de contrastar la situación de la empresa y que es lo que esperan sus integrantes para que sea de utilidad a las empresas estudiadas y determinen los cursos de acción necesarios para lograr las características que requiere la cultura deseada. De tal forma y bajo este contexto surge la interrogante: ¿Cuál es la cultura organizacional dominante y preferida que se identifica en empresas de servicios de Ciudad Victoria, Tamaulipas?

Para este estudio se eligieron seis empresas familiares del giro de servicios de Ciudad Victoria Tamaulipas. Cada empresa se identificó con uno de los tipos de cultura propuestos por Cameron y Quinn (1999), descrito en la sección de metodología.

Con la presente investigación se propone aportar evidencia empírica sobre las características de la cultura organizacional de las empresas familiares y cómo se establece tal relación en el giro de servicios, que puede ser diferente en empresas de diferente actividad, como la industrial y comercial, en las que los productos son tangibles. Esta investigación también abona al estado de la investigación en México sobre la empresa familiar, que se encuentra en proceso de desarrollo (Soto, 2013).

De igual forma, la aportación que se busca con el estudio es concientizar a los empresarios, sean dueños de empresas familiares o no familiares, sobre la importancia de identificar las características de la cultura organizacional que se presenta en sus organizaciones, puesto que son la base para comprender el comportamiento de sus integrantes y conducirlos hacia el logro de los objetivos organizacionales, generando el cambio que se requiera en la conducta de los mismos, buscándose en todo momento el bienestar y satisfacción del trabajador, dado que existe sustento teórico de que determinadas características de la cultura organizacional va a generar bienestar y satisfacción laboral (Calderón, et. al., 2003). La identificación del tipo de cultura organizacional de la empresa familiar permite a los tomadores de decisiones de la misma, tener las bases necesarias para tomar las decisiones pertinentes que contribuyan a lograr una ventaja competitiva para su permanencia en el mercado, de tal manera que se pueda reducir el alto porcentaje de empresas que fracasan, porque refuerza el sentido de pertenencia y contribuye a desarrollar estrategias para su sostenibilidad (Alvarado y Monroy, 2013). 


\section{MARCO TEÓRICO}

La cultura organizacional es un concepto inherente a la teoría del comportamiento organizacional, derivada de la escuela de las relaciones humanas. Los elementos que conforman la cultura organizacional se constituyen en valores, creencias, hábitos, costumbres, tradiciones, entre otros que permiten caracterizar la conducta de los integrantes de la organización en su conjunto.

La cultura organizacional se traduce en comportamientos en la empresa. La conducta, actitudes y el comportamiento, en general, de los integrantes de la organización están determinados por las creencias, valores, hábitos y demás características que la definen, pero en el caso de la empresa familiar, estos elementos provienen principalmente de los fundadores.

Existen diversas y diferentes definiciones de cultura organizacional, Cújar (2013) la define como "el conjunto de significados compartidos y creencias en poder de una colectividad", las características que la distinguen son la regularidad en los comportamientos observados, las normas, los valores dominantes en la empresa, su filosofía, sus reglas y el clima de la organización.

En general, los comportamientos derivados de la cultura organizacional se traducen en el clima de la organización, que en la empresa familiar adopta características distintivas por la participación del propietario y sus descendientes, ya que en ellas la participación de la familia y el tiempo que la misma le dedica son factores clave para ser consideradas como tal.

La empresa familiar presenta particularidades: los valores que prevalecen en la organización son transmitidos por el fundador o fundadores, la participación de miembros de la familia determina en gran medida las formas de llevar a cabo los procesos organizacionales.A diferencia de la empresa no familiar, en la que la cultura organizacional se forma con la interacción de los integrantes de todos los niveles jerárquicos, en la familiar, la principal responsabilidad de definir una cultura organizacional con características que le permitan la permanencia en el mercado es de los fundadores.

Los miembros de la organización en la empresa familiar muestran especial atención a la conducta del fundador o de los familiares del dueño para adoptar con base a ello su conducta y actitud.

La cultura organizacional de la empresa familiar ha sido estudiada por diversos autores, en distintos países y a través de diferentes modelos. Los modelos que permiten diagnosticar, evaluar y medir el tipo de cultura organizacional que prevalece en las empresas se pueden observar en la Tabla 1. El modelo teórico que sustenta este trabajo es el desarrollado por Cameron y Quinn y los dos factores considerados para la definición de empresa familiar son los señalados por Leach (2009) citado por Garza et. al. (2011). 


\begin{tabular}{|c|c|c|c|}
\hline Modkls & Aainr & Ane & Deacripsias \\
\hline $\begin{array}{l}\text { Mndein da } \\
\text { nevioma }\end{array}$ & \begin{tabular}{|l|} 
Tes y \\
Dentivese
\end{tabular} & 31015 & 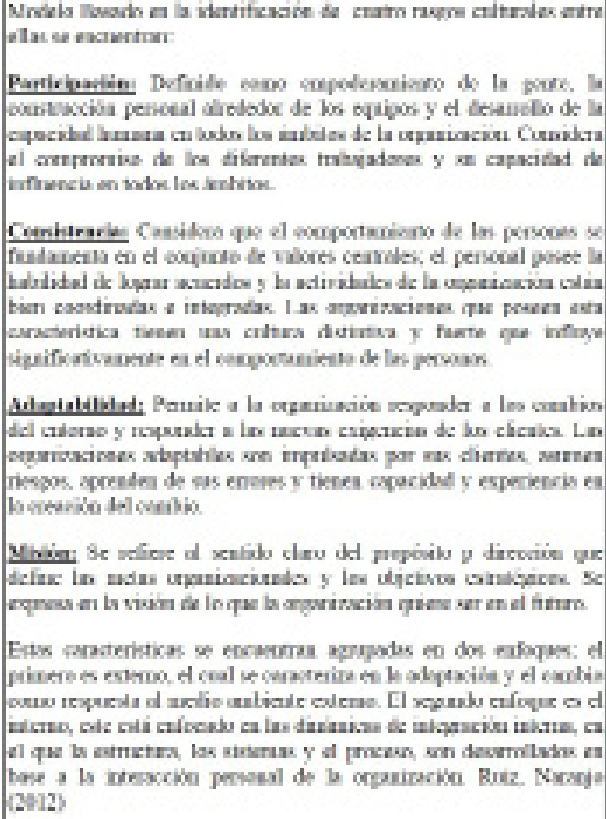 \\
\hline $\begin{array}{l}\text { Slodelo } \\
\text { Oegrivarreal } \\
\text { Cinure Profle }\end{array}$ & \begin{tabular}{|l|} 
Oracily \\
Croman \\
Codwel
\end{tabular} & 1901 & 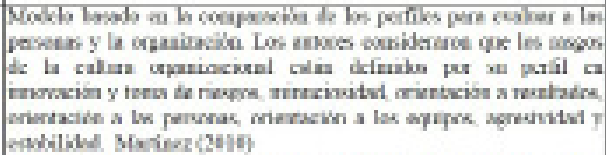 \\
\hline $\begin{array}{l}\text { Sindein de la } \\
\text { Cinco } \\
\text { Disclsioses }\end{array}$ & Ifrefinede & $19 m$ & 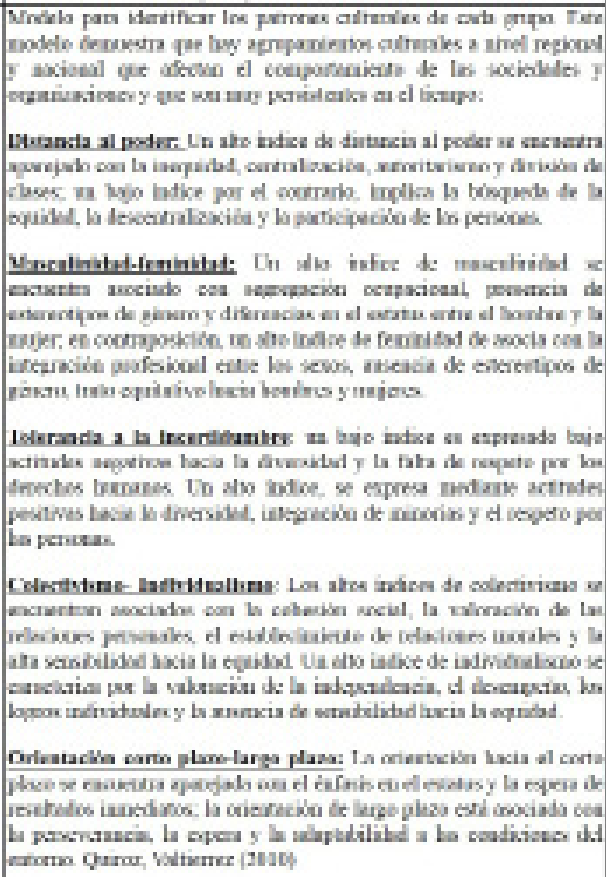 \\
\hline 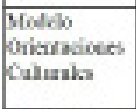 & Heniset & 1972 & 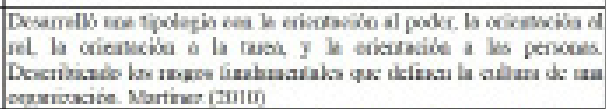 \\
\hline $\begin{array}{l}\text { Canpeting } \\
\text { Valvs } \\
\text { Francwok } \\
\text { CVVF) }\end{array}$ & \begin{tabular}{|l|} 
ranem \\
cy \\
coine
\end{tabular} & 1900 & 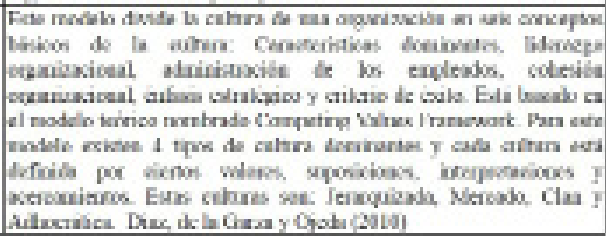 \\
\hline
\end{tabular}

Tabla 1. Modelos de Cultura Organizacional
En el caso particular de este estudio se ha considerado oportuno el utilizar la metodología propuesta por Cameron y Quinn (1999). Estos autores se basan en el "Competing Values Framework" propuesto por Quinn (1998), para crear lo que se denomina "Instrumento para la valoración de la cultura organizacional (Organizacional Cultural Assessment Instrument, OCAI)" este mide la situación cultural de la empresa sobre cuatro dimensiones de cultura (Tabla 2).

\begin{tabular}{l|l}
\hline & $\begin{array}{l}\text { La empresa se concibe como una gran familia, donde sus miem } \\
\text { comparten interactivamente creencias y valores. }\end{array}$ \\
\hline lica & $\begin{array}{l}\text { La organización se concentra en posicionarse externamente con un } \\
\text { grado de flexibilidad y de individualidad. }\end{array}$ \\
\hline ada & $\begin{array}{l}\text { La organización se concentra en lograr posicionarse extremadam } \\
\text { con la necesidad por estabilidad y control, orientada a la consecu } \\
\text { de resultados. }\end{array}$ \\
$\begin{array}{l}\text { La organización se encuentra extraordinariamente normalizadi } \\
\text { cuanto a funciones, responsabilidades, procesos, procedimie } \\
\text { tareas y actividades, remarcando de algin modo su cari } \\
\text { burocrático. }\end{array}$
\end{tabular}

Tabla 2. Clasificación de cultura organizacional de la empresa familiar

Cada tipo de $\begin{array}{r}\text { cultura } \\ \text { organizacional }\end{array}$
presenta
particularidades, cuál predomine presentará a la vez ventajas y desventajas, que beneficios les represente dependerá de los objetivos que se haya planteado la organización. 
Estudios recientes reconocen que el sector servicios ha ganado importancia en la economía de México, de hecho, las actividades del sector terciario rebasan, por mucho, las actividades del sector secundario en cuanto a la contribución que reportan al producto interno bruto (PIB) y la generación de empleo. Además los servicios contribuyen con aproximadamente $60 \%$ a la producción agregada, mientras que el sector secundario representa cerca de $30 \%$ (Flores, et. al. 2013).

Para Tamaulipas, el Producto Interno Bruto (PIB) del estado ascendió a más de 458 mil millones de pesos en 2013, con lo que aportó 3.0\% al PIB nacional. Las actividades terciarias, entre las que se encuentran el comercio y servicios inmobiliarios, aportaron $61 \%$ al PIB estatal en 2013 (PROMEXICO (2013).

El modelo de Cameron y Quin (1999), sustento de este estudio, busca la evaluación de seis dimensiones dentro de la cultura organizacional basada en: a) características dominantes, b) liderazgo organizacional, c) administración de recursos humanos, d) unión de la organización, e) énfasis estratégicos y f) criterios de éxito, que en conjunto determinan la cultura organizacional de las empresas analizadas.

Por su aportación a la economía nacional, se seleccionó el giro de servicios para realizar el análisis. Además, de las empresas familiares establecidas en Cd. Victoria, Tamaulipas predominan las de este sector. La Tabla 3 concentra las características esenciales de las empresas objeto de estudio.

\section{Metodologia}

\begin{tabular}{l|c|c|c|r}
\hline $\begin{array}{l}\text { umero de } \\
\text { Empresa }\end{array}$ & Servicio & $\begin{array}{c}\text { Años en el } \\
\text { mercado }\end{array}$ & $\begin{array}{c}\text { Número de } \\
\text { empleados }\end{array}$ & $\begin{array}{r}\text { Clasificación } \\
\text { empresa, seg } \\
\text { número de emp } \\
\text { SIEM (201. }\end{array}$ \\
\hline impresa 1 & Ferretería & 5 años & 10 & Micro \\
\hline impresa 2 & Constructora & 9 años & 117 & Mediana \\
\hline impresa 3 & Óptica & 17 años & 5 & Micro \\
\hline
\end{tabular}

Tabla 3. Empresas familiares objeto de estudio

La estructura metodológica se resume en las fichas técnicas de las Tablas 4 y 5 . El enfoque es cuantitativo porque se aplicó el método de encuesta, que permite, a través de la aplicación de un cuestionario estructurado medir las variables involucradas y determinar de qué magnitud son las que integran cada uno de los tipos de culturas organizacionales de la empresa familiar e identificar la que predomina. 


\begin{tabular}{|ll|}
\hline Conceptos & Descripción \\
\hline Enfoque de estudio & Cuantitativo \\
\hline Diseño y tipo de investigación & No experimental- transversal \\
\hline Alcance de la investigación & Descriptivo \\
\hline Método & Encuesta \\
\hline
\end{tabular}

El diseño de la investigación es no experimental, transversal, descriptiva, por el hecho que especifica y detalla la caracterización de la cultura dominante y preferida en las empresas familiares de servicios en Ciudad Victoria Tamaulipas estudiadas.

Tabla 4: Ficha técnica según el tipo de investigación utilizado

La selección se concentró en 6 empresas familiares (Tabla 5), según investigaciones realizadas en la empresa familiar por el método de caso, la mediana representa tres empresas de estudio. El valor de este estudio estaría por encima de este valor. Además las investigaciones realizadas han privilegiado a las pequeñas y medianas empresas familiares. Solamente el $15 \%$ de ellas se han realizado en grandes empresas familiares y $11 \%$ de los casos tratan indistintamente empresas pequeñas, medianas y grandes (Soto, 2013).

\begin{tabular}{|ll|}
\hline Conceptos & Descripción \\
\hline Unidad de Análisis & $\begin{array}{l}\text { Seis Empresas Familiares del giro de } \\
\text { Servicios de Ciudad Victoria Tamaulipas }\end{array}$ \\
\hline Sujetos de análisis & Trabajadores y fundadores \\
\hline Población & $\begin{array}{l}171 \text { total de trabajadores y fundadores } \\
\text { de las seis empresas }\end{array}$ \\
\hline $\begin{array}{l}\text { Instrumento de recolección de } \\
\text { información }\end{array}$ & Cuestionario de Cameron y Quin (1999) \\
\hline
\end{tabular}

Tabla 5. Ficha técnica del instrumento de recolección de información. 
Instrumento de obtención de información

El instrumento de Cameron y Quin (1999) está compuesto por un cuestionario que requiere que los individuos respondan a solo seis preguntas que abarca las seis dimensiones de la Cultura Organizacional donde se identifican; Características Dominantes, Liderazgo Organizacional, Administración de Recursos Humanos, Unión de la Organización, Énfasis Estratégicos y Criterios de Éxito; con cuatro opciones de respuesta cada una dependiendo del grado de similitud que tenga la descripción con la realidad de su propia organización con las letras A cultura Clan, B cultura Adhocrática, C cultura Mercado, y D cultura Jerarquizada.

Los puntajes de cada una de las preguntas son sumados y promediados para obtener un grafico resumen del perfil o caracterización de las empresas familiares estudiadas. El instrumento consta de dos partes, una para evaluar la cultura organizacional actual y otra para evaluar la cultura organizacional deseada por los integrantes de las mismas.

El porcentaje de cuestionarios no válidos ascendióa8\%, porloquedelos 186 cuestionarios aplicados sólo se consideraron para el análisis 171. El porcentaje de cuestionarios no válidos se debió principalmente a información incompleta o sesgada por parte del encuestado, principalmente debido a que el porcentaje por respuesta debe ser máximo 100 según el modelo.

\section{Procedimiento metodológico}

El instrumento utilizado fue aplicado a todo el personal de las empresas familiares incluyendo trabajadores y fundadores, esté requiere que los individuos respondan a solo seis preguntas tipo con cuatro sub alternativas cada una catalogada con las letras A que trata de medir el grado de orientación de la empresa a la cultura Clan, B orientado la cultura Adhocratica, C orientado a medir el grado de orientación a la cultura Mercado, y D orientado a la cultura Jerarquizada. Frente a cada letra, se encuentran a su vez dos columnas, una llamada Actual y otra Deseada en la que cada empleado de las empresas familiares estudiadas debe responder.

En estas columnas el encuestado asigna un puntaje de 1 a 100 puntos entre estas cuatro alternativas de acuerdo a cuan similar es cada una con su empresa, partiendo principalmente de la columna actual que corresponde a la identificación de la Cultura Actual de la empresa. Un puntaje más alto significa mayor afinidad y la suma final del puntaje debe ser 100. Enseguida responde a la columna titulada Deseada, se anotan los puntajes que según el entrevistado debería ser o adoptar la cultura organizacional.

Una vez que se tienen los puntajes en cada columna se procede a calcular el promedio de puntos por cada una de las letras, sumando todos los puntajes, por ejemplo de la letra A y se divide entre seis, se procede a sacar el promedio de las letras restantes B, C, y D. Mientras más cerca al 100 esté una organización es el tipo de cultura predominante en la empresa.

Los resultados obtenidos son plasmados en un eje de coordenada que permite observar gráficamente las características y la orientación de la cultura organizacional por empresa familiar y posteriormente en su conjunto la cultura dominante actual y deseada en las empresas familiares.

\section{ANÁLISIS DE LOS RESULTADOS}

La Cultura organizacional predominante actual en las seis empresas familiares del giro de servicios en Ciudad Victoria Tamaulipas es la que se concibe como una gran familia, donde sus miembros comparten interactivamente creencias y valores, denominada Clan (Tabla 6). Este tipo de cultura tiene la ventaja de que los miembros 
se identifican con la organización, su sentido de pertenencia es mayor, lo que impacta positivamente en su conducta y actitud y ello se traduce en beneficio para el desempeño y logro organizacionales.

Tabla 6. Cultura organizacional actual en las empresas de servicios seleccionadas en Cd. Victoria, Tamaulipas

\begin{tabular}{|l|r|r|r|r|}
\hline $\begin{array}{c}\text { CULTURA } \\
\text { PREDONINANTE } \\
\text { ACTUAL }\end{array}$ & \multicolumn{1}{|c|}{ CLAN } & ADHOCRACIA & MERCADO & JERARQUIZADA \\
\hline Empresa 1 & 25.17 & 13.67 & 32.58 & 28.58 \\
\hline Empresa 2 & 24.05 & 24.32 & 22.90 & 28.74 \\
\hline Empresa 3 & 27.67 & 18.83 & 23.67 & 29.83 \\
\hline Empresa 4 & 28.33 & 20.83 & 22.50 & 28.33 \\
\hline Empresa 5 & 23.67 & 17.00 & 32.67 & 26.67 \\
\hline Empresa 6 & 63.81 & 16.83 & 13.41 & 5.95 \\
\hline PROMEDIO & $\mathbf{3 2 . 1 2}$ & $\mathbf{1 8 . 5 8}$ & $\mathbf{2 4 . 6 2}$ & $\mathbf{2 4 . 6 8}$ \\
\hline
\end{tabular}

En la Gráfica 1 se muestra la cultura dominante actual de las seis empresas familiares del giro de servicios estudiadas. Acorde al resultado del cuestionario aplicado, cuatro de las empresas familiares muestran una cultura actual dominante tendiente a ser jerarquizada.

Según lo anterior y tomando en cuenta el modelo de Cammeron y Quinn el resultado obtenido indica que la organización es un lugar estructurado y formalizado para trabajar, los procedimientos gobiernan y dicen a las personas que hacer en el diario quehacer, asi mismo los propietarios muestran interes y preocupación fundamental por una direccion estable y el funcionamienro eficaz de la organización. Sin embargo se puede observar que la empresa 5 los resultados tienden hacia la cultura mercado, es decir, orientada a los resultados cuya preocupacion es realizar el trabajo bien hecho.

Fuente: Elaboración propia (2015)

Un factor que determina el resultado anterior son los años en el mercado, esta empresa solo tiene dos años relativamente pocos enfocandose presisamente en la busqueda de estabilidad y posisionamiento en el mercado. Sin embargo, la empresa 6 se orienta plenamente a una cultura tipo Clan, que según el modelo de Cammeron y Quinn se considera un lugar amistoso para trabajar como una familia unida por la lealtad o

Gráfica 1. Cultura predominante actual en las empresas familiares de servicios estudiadas. la tradición, un factor determinante son los más de 72 años en el mercado al igual que la empresa 4

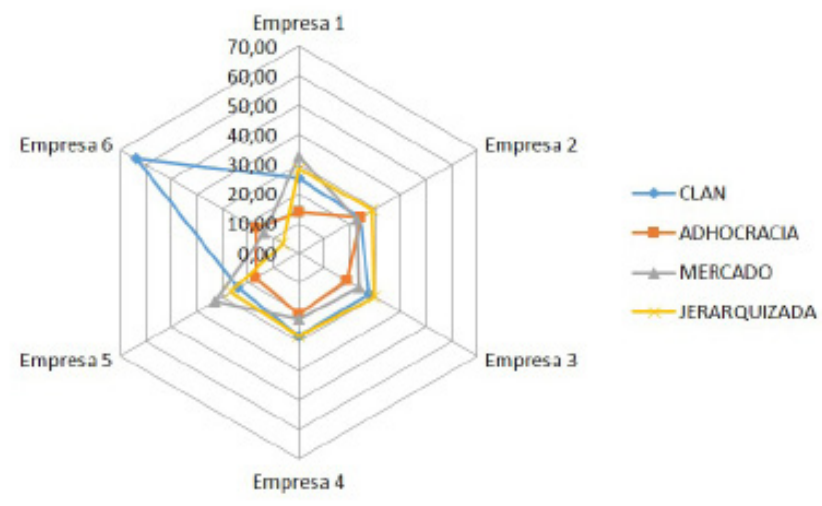

con más de 15 años en el mercado.

En la Gráfica 2 y Tabla 7 se pueden observar las seis preguntas que abarcan las seis dimensiones de la Cultura Organizacional y que individualmente determinan en su conjunto la cultura dominante en cada empresa familiar, se aprecia que para la situación actual la cultura Clan es la que tiene mayor puntación en las seis empresas analizadas con un $32.12 \%$ y en menor grado la cultura

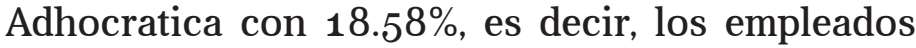
ven la organización como un lugar poco dinámico para trabajar o con espíritu emprendedor. 
Tabla 7. Características de la cultura actual dominante en las empresas de servicios seleccionadas.

\begin{tabular}{|l|r|r|r|r|}
\hline $\begin{array}{c}\text { CARACTERISTICAS } \\
\text { DE LA CULTURA } \\
\text { ACTUAL DOMINANTE }\end{array}$ & CLAN & ADHOCRACIA & MERCADO & JERARQUIZADA \\
\hline $\begin{array}{l}\text { CARACTERISTICAS } \\
\text { DOMINANTES }\end{array}$ & 29.49 & 19.62 & 26.75 & $\mathbf{2 4 . 1 5}$ \\
\hline $\begin{array}{l}\text { LIDERAZGO } \\
\text { ORGANIZACIONAL }\end{array}$ & 30.34 & 19.63 & 21.42 & 28.61 \\
\hline $\begin{array}{l}\text { ADMINISTRACIÓN DE } \\
\text { RECURSOS HUMANOS }\end{array}$ & 36.26 & 16.40 & 23.22 & 24.11 \\
\hline $\begin{array}{l}\text { UNION DE LA } \\
\text { ORGANIZACIÓN }\end{array}$ & 32.19 & 17.99 & 28.01 & 21.83 \\
\hline ENFASIS ESTRATEGICOS & 30.75 & 18.19 & 23.50 & 27.56 \\
\hline CRITERIOS DE ÉXITO & 33.67 & 19.65 & 24.83 & 21.86 \\
\hline PROMEDIO & $\mathbf{3 2 . 1 2}$ & $\mathbf{1 8 . 5 8}$ & $\mathbf{2 4 . 6 2}$ & $\mathbf{2 4 . 6 8}$ \\
\hline
\end{tabular}

Gráfica 2. Características de la cultura actual dominante en las empresas de servicios seleccionadas.

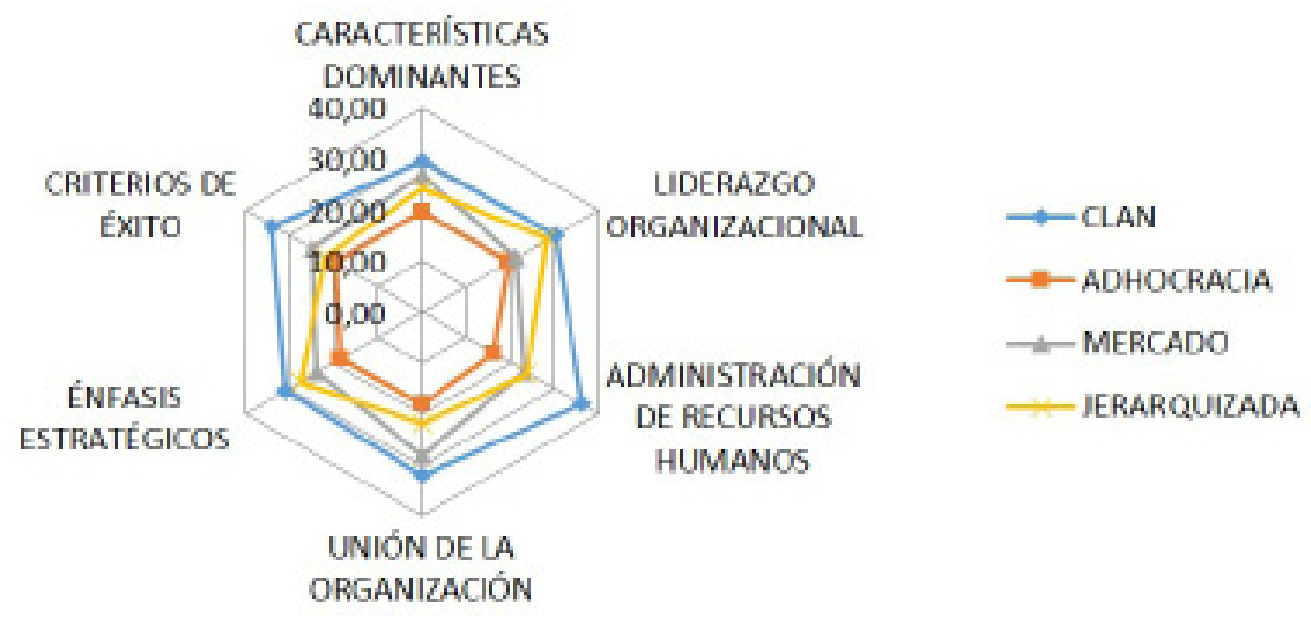

En las empresas estudiadas predomina la cultura organizacional tipo Clan y la que se presenta en menor nivel es la Adhocrática (Tabla 8).

\begin{tabular}{|c|r|r|r|r|}
\hline & CLAN & ADHOCRACIA & MERCADO & JERARQUIZADA \\
\hline $\begin{array}{c}\text { CULTURA } \\
\text { ACTUAL }\end{array}$ & 32.12 & 18.58 & 24.62 & 24.68 \\
\hline
\end{tabular}

Tabla 8. Cultura organizacional actual de las empresas de servicios seleccionadas 
En la Gráfica 3 se muestra el análisis de los promedios para cada tipo de cultura, indica que el tipo de cultura dominante actual en las seis empresas familiares de servicios analizadas es mayormente de tipo Clan seguido de la cultura tipo Jerarquizada, Mercado y en menor grado la Adhocratica. Lo que indica que las empresas familiares estudiadas tienen una cultura organizacional como si fueran una gran familia con valores y metas compartidas entre sus trabajadores, empresas que buscan control interno pero con flexibilidad y preocupados siempre por sus empleados.

\section{CULTURA PREDOMINANTE EN LAS EMPRESAS FAMILIARES ESTUDIADAS}

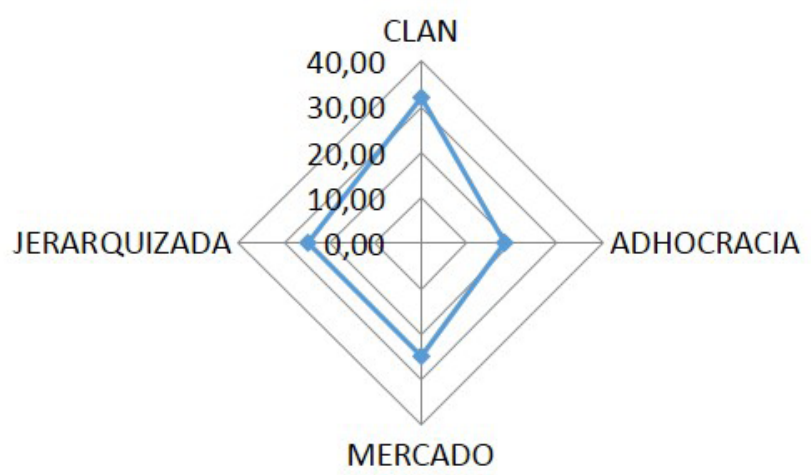

Gráfica 3. Cultura organizacional actual predominante en las empresas familiares de servicios estudiadas

Los resultados obtenidos en la segunda parte de este estudio relacionado a conocer la cultura deseada en las empresas familiares se resumen en la Tabla 9.

\begin{tabular}{|l|r|r|r|r|}
\hline $\begin{array}{l}\text { CULTURA } \\
\text { DESEADA }\end{array}$ & CLAN & ADHOCRACIA & MERCADO & JERARQUIZADA \\
\hline Empresa 1 & 28.83 & 15.92 & 32.17 & 23.08 \\
\hline Empresa 2 & 24.17 & 25.30 & 23.33 & 27.22 \\
\hline Empresa 3 & 33.17 & 23.50 & 21.67 & 21.67 \\
\hline Empresa 4 & 30.71 & 24.33 & 22.90 & 22.14 \\
\hline Empresa 5 & 25.67 & 34.00 & 17.83 & 22.50 \\
\hline Empresa 6 & 64.96 & 18.02 & 11.11 & 5.91 \\
\hline PROMEDIO & $\mathbf{3 4 . 5 8}$ & $\mathbf{2 3 . 5 1}$ & $\mathbf{2 1 . 5 0}$ & $\mathbf{2 0 . 4 2}$ \\
\hline
\end{tabular}

Tabla 9. Cultura organizacional deseada en las empresas de servicios seleccionadas en Cd. Victoria, Tamaulipas. 
Se puede observar también en la Gráfica 4 la cultura predominante deseada en las seis empresas familiares de manera individual, los resultados obtenidos indican que tres de las empresas cambian su percepción a la cultura actual.

\section{CULTURA PREDOMINANTE EN LAS EMPRESAS FAMILIARES ESTUDIADAS}

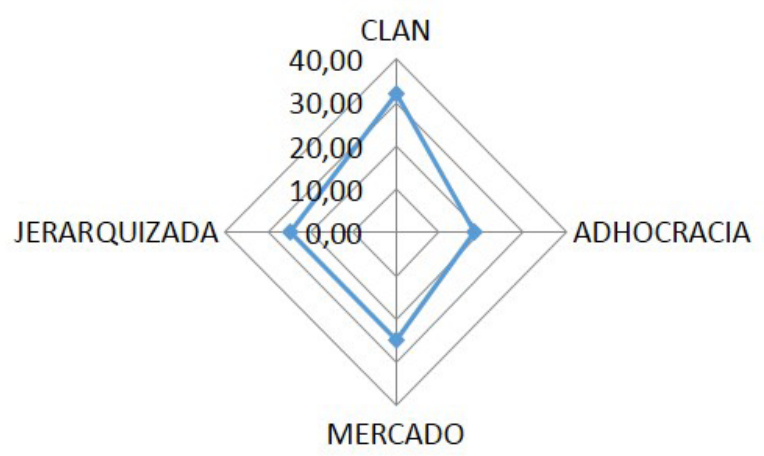

Gráfica 3. Cultura organizacional actual predominante en las empresas familiares de servicios estudiadas

En la empresa 1 su cultura deseada es de tipo Mercado con un $32.17 \%$ y en menor grado la Adhocratica con $15.92 \%$. Manteniendo su percepción de cultura actual con la deseada. La empresa 2 mantiene su cultura actual y deseada de tipo Jerarquizada con $27.22 \%$, así como la empresa 6 mantiene la tipo Clan con $64.96 \%$ y en menor grado la jerarquizada con $5.91 \%$. La empresa 3 y 4 la cultura deseada predomina mayormente la tipo Clan y en menor grado la cultura tipo Jerarquizada siendo esta ultima su cultura actual predominante. La empresa 5 indica el tipo de cultura deseada Adhocratica con un 34\% y en menor grado la tipo mercado con un $17.83 \%$ siendo esta su cultura predominante actual.

Para contrastar la cultura actual de las empresas de servicios analizadas con la cultura deseada, se aplica la misma metodología y los resultados se muestran en la Tabla 10.

\begin{tabular}{|l|r|r|r|r|}
\hline $\begin{array}{c}\text { CARACTERISTICAS DE } \\
\text { LA CULTURA DESEADA } \\
\text { DOMINANTE }\end{array}$ & CLAN & ADHOCRACIA & MERCADO & JERARQUIZADA \\
\hline $\begin{array}{l}\text { CARACTERISTICAS } \\
\text { DOMINANTES }\end{array}$ & 35.98 & 26.44 & 20.29 & 17.29 \\
\hline $\begin{array}{l}\text { LIDERAZGO } \\
\text { ORGANIZACIONAL }\end{array}$ & 31.90 & 22.76 & 26.98 & 18.37 \\
\hline $\begin{array}{l}\text { ADMINISTRACIÓN DE } \\
\text { RECURSOS HUMANOS }\end{array}$ & 36.67 & 20.01 & 20.25 & 23.06 \\
\hline $\begin{array}{l}\text { UNIÓN DE LA } \\
\text { ORGANIZACIÓN }\end{array}$ & 35.88 & 23.00 & 19.64 & 21.50 \\
\hline ÉNFASIS ESTRATÉGICOS & 32.90 & 23.64 & 22.02 & 21.53 \\
\hline CRITERIOS DE ÉXITO & 34.19 & 25.20 & 19.82 & 20.79 \\
\hline PROMEDIO & 34.58 & $\mathbf{2 3 . 5 1}$ & $\mathbf{2 1 . 5 0}$ & $\mathbf{2 0 . 4 2}$ \\
\hline
\end{tabular}

Tabla 10. Características de la cultura deseada dominante en las empresas de servicios seleccionadas. 
En la Gráfica 5 se observa las preguntas que abarcan las seis dimensiones de la Cultura Organizacional que son las características individuales que determinan en su conjunto el tipo de cultura en las seis empresas familiares es tipo Clan con un promedio de $34.58 \%$ y en menor grado la cultura tipo Jerarquizada con un promedio de $20.42 \%$, que de manera individual lo determinan las puntuaciones a las características dominantes con $17.19 \%$, liderazgo organizacional con un $18.37 \%$ y énfasis estratégicos con un $21.53 \%$. A diferencia de las características individuales actuales que en su puntuación menor predomina en las seis empresas la cultura tipo Adhocratica.

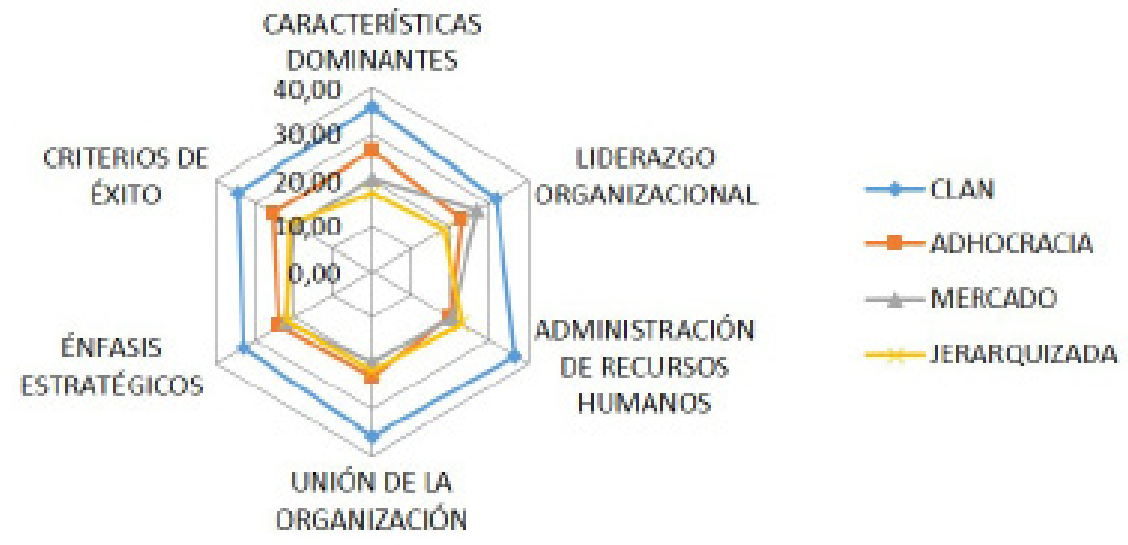

Gráfica 5. Características de la cultura organizacional deseada dominante en las empresas de servicios seleccionadas.

En el análisis de la cultura deseada se obtuvieron resultados diferentes en relación a la cultura actual en tres de las seis empresas (Tabla 11).

\begin{tabular}{|r|r|r|r|r|}
\hline & CLAN & ADHOCRACIA & MERCADO & JERARQUIZADA \\
\hline CULTURA DESEADA & 34.58 & 23.51 & 21.50 & 20.42 \\
\hline
\end{tabular}

Tabla 11. Cultura organizacional deseada en las empresas de servicios seleccionadas

En la Gráfica 6 se muestra los promedios de las características que determinan la cultura deseada de manera general, indica que el tipo de cultura dominante deseado en las seis empresas familiares de servicios es de tipo Clan con un $34.58 \%$ seguido de la cultura tipo Adhocratica con un $23.51 \%$, Mercado con $21.50 \%$ y en menor grado la Jerarquizada con $20.42 \%$. 


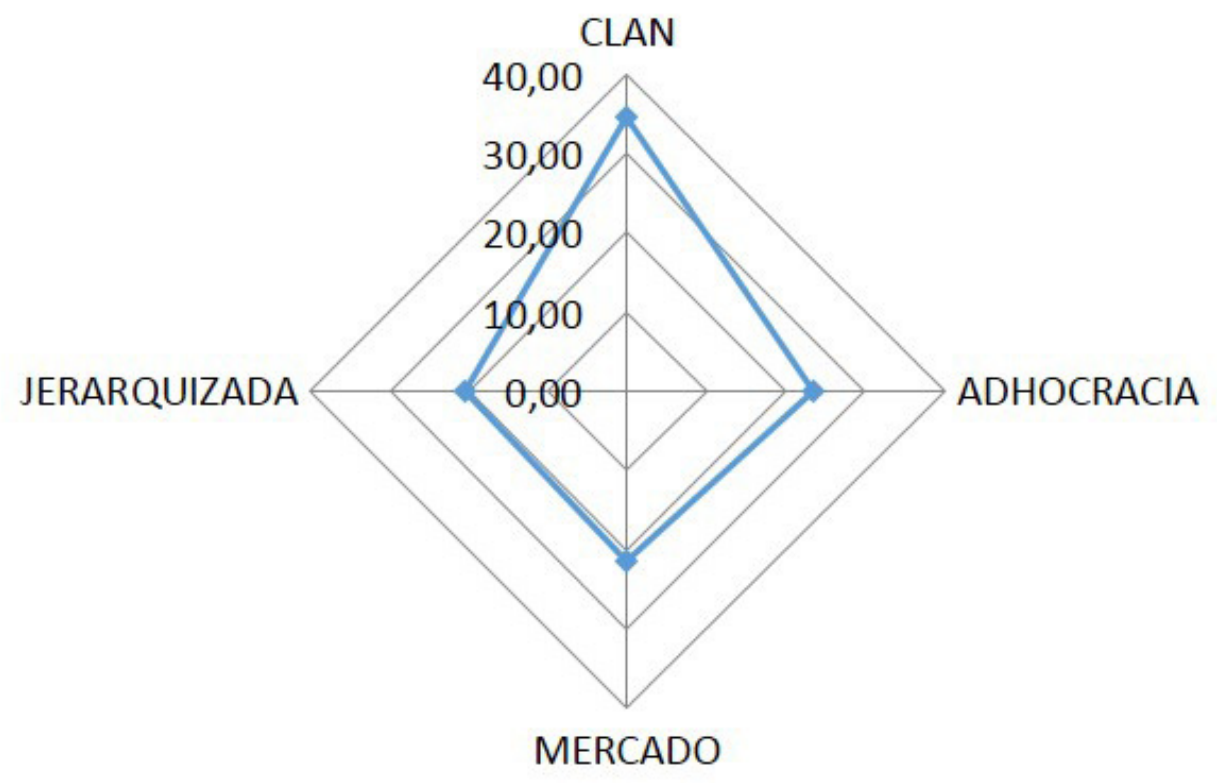

Los resultados comparados de la cultura actual y deseada se resumen en la Tabla 12. La cultura Clan predomina en ambas, pero se presentan cambios importantes en la adhocrática, de mercado y jerarquizada.

\begin{tabular}{|l|c|c|c|c|}
\hline & CLAN & $\begin{array}{c}\text { ADHOCRA- } \\
\text { CIA }\end{array}$ & MERCADO & JERARQUIZADA \\
\hline CULTURA ACTUAL & 32.12 & 18.58 & 24.62 & 24.68 \\
\hline CULTURA DESEADA & 34.58 & 23.51 & 21.50 & 20.42 \\
\hline
\end{tabular}

Tabla 12. Comparación de la cultura organizacional actual y deseada en las empresas de servicios de Cd. Victoria, Tamaulipas seleccionadas.

En la Gráfica 7 se observa una vez obtenidos los resultados que prevalece en la empresa familiar la cultura tipo Clan tanto en la cultura actual con un $32.12 \%$ y en la cultura deseada con un $34.58 \%$, por lo que se puede decir que las empresas familiares buscan propiciar en sus empleados un ambiente de trabajo agradable, que sean consideradas como una familia y que aunque se establezcan reglas, se está abierto a la participación de todos trabajando en equipo. También se puede observar que la cultura actual con menor impacto en la organización es la tipo Adhocracia con un $18.58 \%$, en tanto que en la deseada con menor promedio es la cultura tipo Jerarquizada con un $20.42 \%$. 


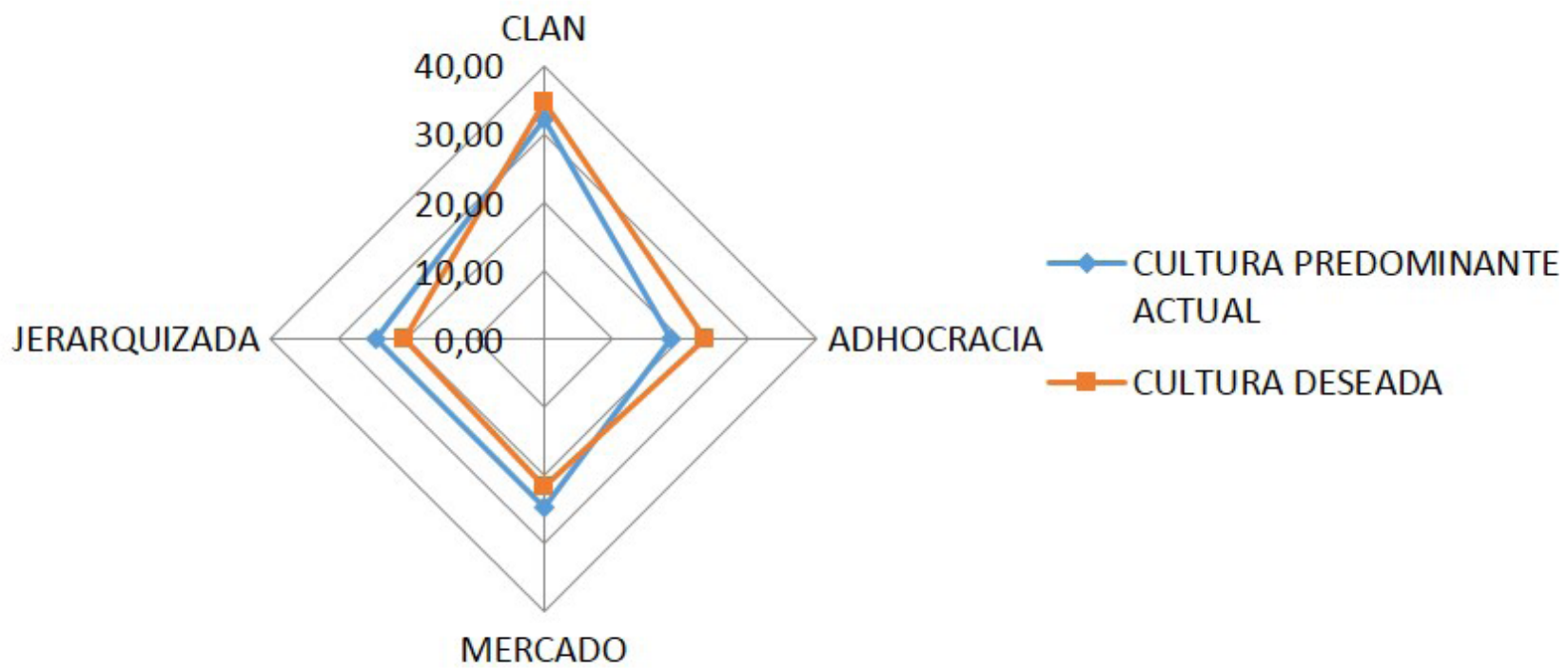

En el estudio de la caracterización de la cultura organizacional actual y deseada en las empresas familiares objeto de análisis, prevalece predominantemente la cultura tipo Clan en ambas, sin embargo si existe cambio de la actual a deseada en las tres restantes, principalmente la cultura tipo Jerarquizada prevalece en último lugar en la cultura deseada, esto indica que los integrantes de las organizaciones no desean trabajar y relacionarse al interior de la empresa por un conjunto de normas y procedimientos rigurosos limitándolos a realizar lo que se les solicita o les indique qué y cómo hacer sus labores. Por el contrario desean una cultura donde la organización sea un lugar dinámico para trabajar, que les permita explotar su espíritu emprendedor con ideas para el fortalecimiento y crecimiento de la organización.

\section{CONCLUSIONES}

El objetivo del estudio se logró al identificar el tipo de cultura que prevalece actualmente y la deseada en las empresas familiares de servicios de la zona de estudio. Queda como evidencia este análisis para futuros trabajos de investigación, entre los cuales se sugiere el estudio de la cultura organizacional en la empresa familiar y la relación con su continuidad.

La cultura dominante actual y deseada en las seis empresas familiares es de tipo Clan, en la que se caracteriza a las empresas familiares estudiadas como un lugar de trabajo muy amistoso donde los trabajadores comparten mucho entre sí, los propietarios se consideran mentores y son figuras paternalistas, está unida por la lealtad o la tradición familiar. En general, el compromiso de su propietario es alto y el éxito empresarial se define en términos de satisfacción al cliente y la participación en equipo.

Cabe mencionar que como resultado de la cultura dominante deseada es la tipo Jerarquizada, lo que significa que los trabajadores encuestados difieren de trabajar y de relacionarse al interior de la empresa por un conjunto de normas y procedimientos que le dicen a ellos que y como hacer sus labores. 
Alvarado Muñoz, Óscar; Monroy del Castillo, Ricardo. 2013. Cultura organizacional en una empresa propiedad de sus trabajadores Cuadernos de Administración, vol. 26, núm. 47, julio-diciembre, 2013, pp. 259-283

Barroso Martínez, A. R. Sanguino Galván y T. M. Bañegil Palacios. 2012. Diferentes criterios del concepto de empresa familiar. Una aportación desde Extremadura, España. Ideas CONCYTEG, 7 83, pp. 611-622

Cameron, Kim \& Quinn, Robert. 1999. Diagnosing and Changing Organizational Culture. Editorial: Addison-Wesley, Series on Organization.

Crespo Casanova, A. J., Bellver Sánchez, A., \& Sánchez y Sánchez, A. M. (2005). La empresa familiar: Manual para empresarios. Barcelona: Deusto.

Cújar Vertel, Angélica del Carmen; Ramos Paternina, Carlos David; Hernández Riaño, Helman Enrique; López Pereira, Jorge Mario Cultura organizacional: evolución en la medición Estudios Gerenciales, vol. 29, núm. 128, 2013, pp. 350-355, https://doi.org/ 10.1016/j.estger.2013.09.009

Esparza Aguilar, José Luis, Domingo García Pérez de Lema, Antonio Duréndez Gómez Guillamón. 2010. La cultura empresarial en la gestión de las empresas familiares: Una aproximación teórica. Investigación y Ciencia. Número 47, pp.13-20
Garza Ramos, María Isabel de la; Medina Quintero, José Melchor; Cheín Schekaibán, Nazlhe Faride; Jiménez Almaguer, Karla Paola; Ayup González, Jannett; Díaz Figueroa, José Guillermo. 2011. Los valores familiares y la empresa familiar en el Nordeste de México. Cuadernos de Administración, vol. 24, núm. 42, enero-junio, 2011, pp. 315-333

Gómez Roldán, Ignacio; Ricardo Bray, Rafael Guillermo. Cultura organizacional: aproximación sectorial en Bogotá Revista Facultad de Ciencias Económicas: Investigación y Reflexión, vol. XX, núm. 2, diciembre, 2012, pp. 19-41, https://doi.org/10.18359/rfce.2162

Soto Maciel, Argentina. La empresa familiar en México. Situación actual de la investigación Contaduría y Administración, vol. 58, núm. 2, abril-junio, 2013, pp. 135-171, https://doi.org/ 10.1016/SO186-1042(13)71213-O

Steckerl Guerrero, Vanessa Modelo explicativo de una empresa familiar que relaciona valores del fundador, cultura organizacional y orientación al mercado Pensamiento \& Gestión, núm. 20, julio, 2006, pp. 194-215 Universidad del Norte Barranquilla, Colombia

Quiroz Guzmán, Tamara; Valtierrez Ángel, David Arnoldo. 2010. Dimensiones Culturales en la empresa estatal de autoservicios, sucursal delicias, Chihuahua. Quinta Época, vol. 27, Julio-enero, 2010, pp. 375-386

Ruiz, Yaz Belinda; Naranjo, Julia Clemencia. 2012. La investigación sobre cultura Organizacional en Colombia: una mirada desde la difusión en revistas científicas. Diversitas: Perspectivas en Psicología, vol. 8, núm. 2, 2012, pp. 285-307, https://doi.org/10.15332/s17949998.2012.0002.06 
Martínez Avella, Mario Ernesto. Relaciones entre cultura y desempeño organizacional en una muestra de empresas colombianas: Reflexiones sobre la utilización del modelo de Denison. Cuadernos de Administración Bogotá Colombia, 23 (40), Enero-junio, 2010, pp. 163-190

Díaz Acevedo, Natalie Berenice; De la garza Carranza, Ma. Teresa; Ojeda Hidalgo, José Felipe. Liderazgo y cultura organizacional en la administración pública en la región laja-bajío. Revista Panorama Administrativo, núm. 8, año 4 (2010). pp. 111- 123.

SIEM (Sistema Empresarial Mexicano). 2016. Directorio de empresas en México. http:// www.siem.gob.mx/siem/portal/consultas/ consulta.asp?q=o Recuperado el 18 de enero de 2016.
Flores Sánchez, Carlos Alberto; Castillo Ponce, Ramón A.; Rodríguez Espinosa, María de Lourdes. La importancia del sector servicios en la economía mexicana: Un análisis de series de tiempo. Paradigma Económico, año 5, núm. 1, Enero-junio 2013. pp. 5-27

Soto Maciel, Argentina. La empresa familiar en México. Situación actual de la investigación. Contaduría y administración, vol. 58, núm. 2, abril-junio, 2013, pp. 135-171. ISBN 0186-1042. Universidad Nacional Autónoma de México, https:// doi.org/10.1016/So186-1042(13)71213-O

PROMEXICO (Secretaria de Economía) 2016. http:// mim.promexico.gob.mx/documentos/PDFl/mim/ FE_TAMAULIPAS_vf.pdf. Recuperado el 18 de enero de 2016.

Copyright (c) 2016 Daniela Cruz Delgado y Estela Torres Ramírez

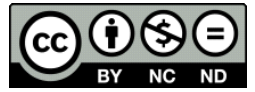

Este texto está protegido por una licencia Creative Commons 4.0

Usted es libre para Compartir (copiar y redistribuir el material en cualquier medio o formato), siempre que cumpla las condiciones de:

Atribución: Usted debe dar crédito a la obra original de manera adecuada, proporcionar un enlace a la licencia, e in dicar si se han realizado cambios. Puede hacerlo en cualquier forma razonable, pero no de forma tal que sugiera que tiene el apoyo del licenciante o lo recibe por el uso que hace de la obra.

No Comercial: Usted no puede hacer uso de la obra con propósitos comerciales

Sin Derivadas: Si remezcla, transforma o crea a partir de la obra, no podrá distribuir la obra modificada 